\title{
REVIEW
}

\section{Gene therapy in autoimmune, demyelinating disease of the central nervous system}

\author{
David Baker and DJR Hankey \\ Institute of Neurology, University College London, UK
}

\begin{abstract}
Multiple sclerosis (MS) is an immune-mediated disease of the central nervous system (CNS), where suspected autoimmune attack causes nerve demyelination and progressive neurodegeneration and should benefit from both antiinflammatory and neuroprotective strategies. Although neuroprotection strategies are relatively unexplored in $M S$, systemic delivery of anti-inflammatory agents to people with MS has so far been relatively disappointing. This is most probably because of the limited capacity of these molecules to enter the target tissue, because of exclusion by the bloodbrain barrier. The complex natural history of MS also means that any therapeutic agents will have to be administered long-
\end{abstract}

term. Gene therapy offers the possibility of site-directed, long-term expression, and is currently being preclinically investigated in experimental autoimmune encephalomyelitis (EAE), an animal model of MS. While some immune effects may be targeted in the periphery using DNA vaccination, strategies both viral and nonviral are being developed to target agents into the CNS either via direct delivery or using the trafficking properties of cell-carrier systems. Targeting of leucocyte activation, cytokines and nerve growth factors have shown some promising benefit in animal EAE systems, the challenge will be their application in clinical use. Gene Therapy (2003) 10, 844-853. doi:10.1038/sj.gt.3302025

Keywords: Autoimmunity; cytokines; experimental allergic encephalomyelitis; gene therapy; multiple sclerosis; neurodegeneration

\section{The Disease Processes in CNS autoimmunity}

Multiple sclerosis (MS) is the major demyelinating disease of the central nervous system (CNS), which is associated with blood-brain barrier (BBB) dysfunction and mononuclear cell infiltration of the white matter. These lesions expand to leave a trail of demyelination and axonal loss that impair neurotransmission, and produce a spectrum of troublesome symptoms. ${ }^{1,2}$ Although MS may follow a variety of unpredictable courses, the typical feature is relapsing-remitting neurological attacks with intermittent and variable recovery followed by a progressive phase where disability continues to steadily worsen over time. ${ }^{1,2}$ MS is a complex polygenic trait, but so far the only identified susceptibility loci are within the major histocompatibility complex (MHC). ${ }^{1,3}$ These antigens are involved in immune-recognition and underscore the pathological and clinical evidence that disease is due to the activities of the immune system (Figure 1). The current thought is that MS occurs because of an autoimmune attack of CNS myelin and oligodendrocytes, which is probably triggered by viral or other environmental microbes. ${ }^{4-6}$

The autoimmune theory is further supported by the observation that similar clinical and histological disease can be induced in a variety of animals by induction of autoimmunity to a number of myelin antigens, including proteolipid protein (PLP), myelin basic protein (MBP) and myelin oligodendrocyte glycoprotein (MOG). ${ }^{1,4,5,7}$

Correspondence: Dr D Baker, Department of Neuroinflammation, Institute of Neurology, University College London, 1 Wakefield Street, London WC1N 1PJ, UK
These animal models develop experimental allergic encephalomyelitis (EAE). ${ }^{7}$ Here, it is clear that autoreactive $\mathrm{T}$ cells provide the organ-specificity of disease. ${ }^{8}$ They initiate a dynamic cascade of proinflammatory and regulatory events that ultimately produce a myelinotoxic microenvironment with eventual axonal destruction ${ }^{1,2,9-}$ 16 (Figure 1). Despite some limited success, disease is poorly managed and there is a real need for effective treatment. $^{1,2}$ Dissection of EAE has played an integral part in defining immune effects that may occur in MS (Figure 1) and, particularly, the development of the Th1/ Th2 paradigm, which is integral to the design of many therapeutic approaches to autoimmunity. ${ }^{17}$ Myelin-specific Th1 cells clearly transfer disease in rodents and nonhuman primates and inhibition of the generation of Th1 responses inhibits disease development. ${ }^{18-21}$ Furthermore, generation of myelin-reactive Th2/Th3 cells can suppress the emergence of pathogenic Th1 activity in EAE. ${ }^{19,22-25}$ However, the therapeutic use of Th2 cytokines (IL-10, TGF $\beta$ ) or use of cytokine agents with an anti-inflammatory profile (IFN $\beta, \mathrm{TNF} \alpha$ receptor $\mathrm{p} 55-\mathrm{Ig}$ ) in MS patients has so far been mostly disappointing. ${ }^{26-30}$ CNS inflammation is shielded from the systemic circulation by the activities of the $\mathrm{BBB},{ }^{31}$ which even if compromised during disease still provides a barrier relative to peripheral sites. ${ }^{9}$ In addition, excessive systemic cytokine release or inhibition will activate homeostatic feedback systems that may further limit clinical utility, and the widespread expression of cytokine receptors may lead to unwanted side-effects becaue of activities outside the CNS. ${ }^{27}$ Owing to the chronic nature of the disease, therapy will need to be prolonged. ${ }^{1,2}$ Gene therapy offers the potential to deliver 


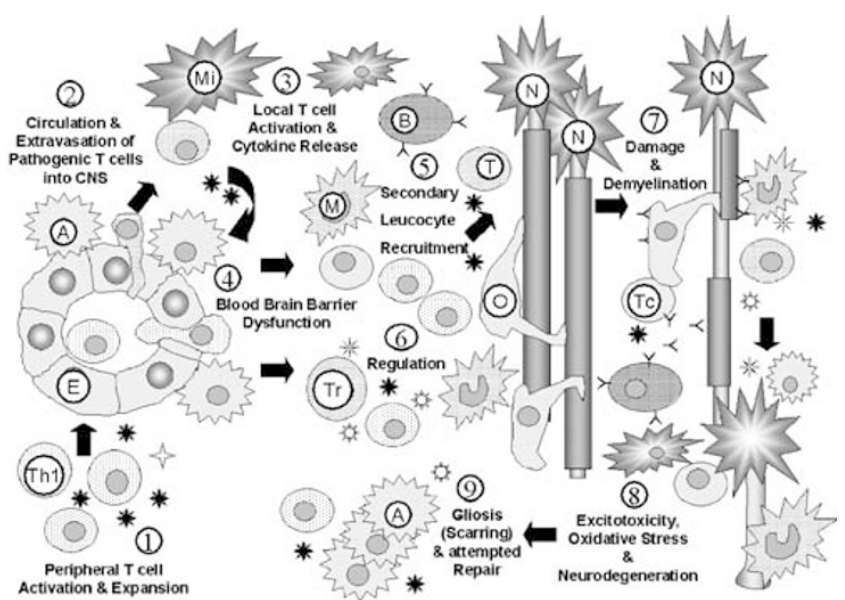

Figure 1 Multiple disease processes occurring in EAE and MS. Tcells (T) are expanded in peripheral lymphoid tissues and once activated they upregulate adhesion molecules (Eg CD49d), immunological receptors and migrate into tissues. If they enter the CNS and encounter antigen presenting microglial (Mi) cells within the perivascular space, they are activated to release proinflammatory cytokines such as IL-1, TNF $\alpha, I F N \gamma$, which activate CNS endothelia (E) to upregulate adhesion molecules (CD54, CD105) and chemokines (MCP-1, MIP-1 $\alpha)$ that facilitate BBB breakdown. This allows a secondary wave of recruitment of monocytes and $T$ and $B$ cells into the perivascular space, and the lymphocytes and macrophages $(M)$ then migrate out into the parenchyma. These produce a variety of cytokines (stars) and they will have proinflammatory and regulatory activities, some of which will be produced by regulatory T cells (Tr, such as Th2/Th3 cells). These will act on infiltrating cells and resident glia and the outcome will depend on the balance of proinflammatory and regulatory molecules. The proinflammatory lesion promotes myelin destruction and death of oligodendrocytes (O). This may occur through oxidative stress, cytokine attack, for example, membrane TNF $\alpha$ and possibly cytotoxic T cells (Tc). Myelin-specific antibodies $(Y)$ will cause destruction of myelin through complement-mediated lysis or by opsonization to promote macrophage engulfment of myelin. Demyelinated nerves must redistribute ion channels to promote some form of normal function and these may be particularly vulnerable to oxidative stress, and excitotoxic damage through glutamate release either from the nerves themselves or from the infiltrating cells and neurons $(N)$. These slowly die and cause the irreversible accumulation of disability. During the process of damage repair, processes are generated which promote development of glial progenitors and remyelination. However, this is eventually abortive and the CNS is scarred by an astrocytic gliosis, which provides a barrier to further repair. ${ }^{1}$

agents long-term, especially within the CNS, to multifocal lesions.

\section{Gene therapy in autoimmune demyelinating disease}

The key issues for any successful gene therapy approach is the nature of the vector and definition of targets. Gene therapy has not yet been attempted in MS, but there have been a number of studies in EAE that have invariably shown some level of efficacy at inhibiting the disease (Table 1), although in many cases this has only been an amelioration rather than elimination of disease. ${ }^{32-64}$ As the majority of the CNS is postmitotic, this puts constraints on the nature of the vector that can be used, and to date administration of plasmid DNA, ${ }^{32-41}$ viral infection, ${ }^{42-53}$ and retrovirally transduced cell (RVC)carriers $^{47,54-63}$ have been investigated in EAE (Table 1). These have largely focused on inhibition of the immune response either applied centrally to target the local pathological events within the CNS or peripherally administered to inhibit: initial sensitization, the activities of circulating cells or perivascular events in areas of local BBB breakdown. In addition, some studies have attempted to promote repair or inhibition of the demyelination process. ${ }^{40,44,49,57,61}$ Cytokines are dynamically expressed as lesions evolve and resolve $\mathrm{e}^{12-16}$ and are of major importance in the development and control of autoimmunity. ${ }^{12}$ Many studies in EAE focus on the use of knockout mice. ${ }^{22,24,65}$ However, in these mice there is cytokine redundancy, compensation and sometimes lethality due to developmental effects. Exogenous gene delivery provides a useful tool to probe the biology of disease in 'physiologically normal' adult animals. Importantly, it also provides a route for therapy, particularly as gene delivery of cytokines can be shown to be more efficacious than bolus protein delivery.

\section{Local immunogene therapy in CNS autoimmunity}

Lesions in MS and EAE occur throughout the $\mathrm{CNS}^{1,2}$ and as there is limited parenchymal diffusion, ${ }^{34}$ this means that the protein would have to be delivered by multiple, invasive injections, which is impractical. This can be overcome by delivery, such that therapeutic agents 'bathe'the CNS through the cerebrospinal fluid (CSF) ${ }^{64}$ Currently, CNS delivery of drugs is achieved through the use of osmotic pumps that are expensive and cumbersome. ${ }^{66}$ Gene therapy offers particular promise in this area. ${ }^{64}$ Where direct comparisons of delivery of inhibitory cytokine and cytokine inhibitory gene vectors have been made, central (CNS) administration exhibits greater efficacy than systemic delivery. ${ }^{34,46,47,54}$ Importantly, the nature of the BBB limits not only influx but also importantly egress of molecules from the brain and can, depending on the dose injected, ${ }^{43,46,47}$ create a local concentration gradient in the CNS that can achieve local therapy, but does not induce circulating levels that cause peripheral suppression of immune responses. ${ }^{47,64}$ Therefore, the CNS may be a unique tissue to deliver potent immunosuppressive agents ${ }^{43}$ (e.g. CTLA4-Ig) that may not be tolerated if delivered to other tissue sites because of the unwanted effects of generalized immunosuppression, such as the development of infection.

Injection of naked plasmid DNA, even following incorporation into cationic lipid, exhibits exceedingly low and transient expression in the CNS. ${ }^{34}$ In contrast, replication-deficient viral vectors such as adenoviral (AV) and herpes simplex viral (HSV) vectors, which can infect postmitotic cells, can reliably produce secreted protein. Intracisternal ${ }^{50-53}$ or intraventricular ${ }^{46}$ delivery of viral vectors shows significant and efficient transduction of the ependymal cell layer that surrounds the brain ventricles and spinal canal. ${ }^{64}$ The intracisternal (ependymal) delivery also targets the choroidal and leptomeningial cells coating the brain and spinal cord and is therefore a useful target for delivering soluble molecules that consistently reaches all CSF spaces and avoids infection of neurons. ${ }^{64}$ Viral vectors can easily produce transgene levels in the range of $10-100 \mu \mathrm{g} / \mathrm{ml}$ within the $\mathrm{CSF}^{43,47,64}$ Expression of these vectors vary from a number of days (AV vectors), ${ }^{43,46,47}$ to about a month (HSV-vectors), ${ }^{64,67}$ which provides sufficient time to undertake experiments in EAE (Table 1). This compares well with the $10-100 \mathrm{ng} / \mathrm{ml}$ often produced for months 
by retrovirally transduced cell vectors. ${ }^{47,54}$ However, the level can be dose-titrated depending on the vector used, and the optimal levels for different cytokines are likely to be varied. Many of the immunological approaches used in gene therapy have evolved around the Th1/Th2 paradigm and IL-4 has been found most consistently to promote inhibition of EAE (Table 1). Furthermore, a HSV-IL4 vector injected into the CNS has been translated from rodents to non-human primates. ${ }^{48,51,53}$ This is important in terms of clinical translation as primates have an immune system more closely resembling that in humans and disease often takes some time to develop, as compared to that in rodents. ${ }^{20}$ When directly compared, IL-4 is generally more effective than IL-10.34,42,50,56 However, notably for IL-10, results have been variable (Table 1). The CNS delivery of high-titre adenoviral human IL-10 could inhibit CNS infiltration and importantly the development of relapsing mouse EAE when administered during remission. ${ }^{46}$ Interestingly, an adenoviral mouse IL-10 (mIL-10) vector, albeit injected at lower titres, was clinically ineffective despite the production of CSF IL-10 levels that compared well with the levels produced by a retroviral cell vector (RCV-mIL10) that inhibited the severity of clinical signs. ${ }^{47}$ The level of infiltration generally correlates with the clinical severity of EAE. ${ }^{68}$ Interestingly, in contrast to the prevention of leucocyte accumulation in the CNS that occurs in most other gene therapy studies, RCV-mIL-10treated animals exhibited significant CNS infiltration but there was a dramatic shift in the phenotype of the infiltration. ${ }^{47}$ This suggests not only the nature of the gene product that can influence outcome, but also the nature of the vector and whether the cell is infected or transfected, possibly through the cytokines they coproduce. ${ }^{69-71}$ There have been few cases in current EAE studies (Table 1) where the biological effect was directly attributed to the secretion of the transgene, ${ }^{38,46,58}$ which makes it difficult to determine the real therapeutic potential of a particular gene product.

While viral vectors are excellent for the transfer of gene products into the CNS, there may be consequences of such direct infection. The CNS exhibits some characteristics of 'immune privilege' that allow potentially immunogenic allogeneic cell donors or viral vectors to be delivered and ignored, thus allowing long-term CNS expression. ${ }^{9,31,72,73}$ However, once the peripheral immune response to the vector/transgene is primed, lymphocytes can cross the BBB, enter the CNS and eliminate the gene vector/product, which can cause additional damage, unless the gene agent protects the host cell. ${ }^{73-75}$ Furthermore, within the context of an inflammatory disease such as MS or EAE once initiated, the pre-existing lesions and BBB dysfunction mean that the immune system is resident and active in the CNS and thus the probability that potentially immunogenic vectors will be recognized is enhanced. ${ }^{1,2,9}$ The immunogenic nature of first generation adenoviral and vaccinia virus vectors is now recognized and 'gutless' vectors have been constructed, which will limit this potential and aid longer-term expression. ${ }^{75}$ Using RCV, the nature of the infected cell is controlled and primary cultures and conditionally (temperature-sensitive) immortalized cells have been used as biological pumps, ${ }^{47,54}$ and have been therapeutically active in relapsing disease. ${ }^{54}$ These RCV can be fully characterized in vitro prior to in vivo analysis.
Initial studies utilized fibroblasts, ${ }^{54}$ as these cells can easily be obtained from any recipient. However, CNSderived cells are more likely to survive in the CNS microenvironment and could also contribute to the repair process. Future studies may consider the use of astrocytes, oligodendrocytes or their precursors, stem cells or Schwann cells. ${ }^{76-78}$ These latter cells can invade the CNS in both EAE and MS and induce remyelination. ${ }^{1}$ These cells produce peripheral myelin, which lacks PLP and MOG that are found in CNS myelin, ${ }^{1}$ and thus may be useful as it may not simply replace the target antigen for the immune response. However, there are major obstacles to migration of cells through the CNS, particularly following the generation of gliotic scars. ${ }^{79,80}$ Clinical Schwann cell transplantation is being examined in MS and likewise, cellular-based biological pumps have been examined in other human CNS diseases. While implantation into the CNS raises safety concerns, such as uncontrolled growth, if the therapeutic activity comes from the action of a biological pump, then it is possible to encapsulate the cells. ${ }^{81}$ This allows even the use of xenogeneic tissue that can be implanted intrathecally via lumbar puncture into the CSF space. Cells are then maintained within the semipermeable membranes to release their products. ${ }^{81}$ Importantly, these could be removed should untoward effects occur.

Systemic immunogene therapy in CNS autoimmunity Systemic gene therapy in EAE has been used in a number of instances, but has typically been applied before or during initial sensitization (Table 1). In these instances, the probable major target is the initial lymphocyte activation that occurs in lymphoid tissue, rather than events within the CNS. Systemic administration of cytokine DNA plasmids has on the whole exhibited no or marginal therapeutic effects (Table 1). Injection of plasmid DNA exhibits poor transduction efficiencies and when used successfully, this has used multiple injections often in regenerating (muscle) tissue. ${ }^{32,33,35-40}$ The regulatory sequences of plasmid DNA is important for efficacy ${ }^{40}$ and these (eg CG repeats) can modulate cytokine production in vivo and can either suppress or worsen EAE, probably because of a secondary effect on sensitization. ${ }^{69,70}$ As an alternative approach to achieving local CNS delivery, the migratory potential of primed T lymphocytes has been harnessed and thus allows for systemic delivery. ${ }^{8}$ The majority of T cells within inflammatory lesions are probably not CNSspecific but are secondarily recruited to the inflammatory site because of their adhesion molecule and chemokine receptor phenotype. ${ }^{10}$ These cells will be targeted to lesional areas and will be activated/maintained locally if they have specificity for myelin or other CNS antigens (Figure 1). T cells can be expanded, infected with replication-deficient retroviral constructs and selected in vitro prior to their systemic delivery. ${ }^{55-61}$ These can even be tracked using coexpression of a marker protein and can be shown to enter lymphoid tissues as well as the CNS. ${ }^{8,82}$ Through the local production of antagonizing molecules in situ they can then inhibit neighbouring pathogenic cells via a bystander effect through release of immunosuppressive and neuroprotective growth factors (Table 1) $)^{55-61}$

Early studies used T-cell hybridomas as cell vectors and showed disease ameliorating potential, but these 
Table 1 Gene therapy vectors used in experimental CNS autoimmunity

\begin{tabular}{|c|c|c|c|c|c|c|c|}
\hline Transgene & Delivery route & Gene vector & Animal strain tested & Inducing antigen & Therapeutic effect & Clinical efficacy & Refs \\
\hline IL-1 $\beta$ & Systemic (iv) & Vaccinia virus & SJL mouse & $\mathrm{SCH}$ & Prophylactic & + & 42 \\
\hline IL-2 & Systemic (iv) & Vaccinia virus & SJL mouse & $\mathrm{SCH}$ & Prophylactic & + & 42 \\
\hline IL-4-Ig & Systemic (im) & Naked DNA & SJL mouse & $\mathrm{MBP}$ & Prophylactic & + & 35 \\
\hline \multirow[t]{10}{*}{ IL-4 } & Systemic (im) & Naked DNA & Lewis rat & MBP peptide & Prophylactic & $=$ & 36 \\
\hline & Systemic (im) & Naked DNA & SJL mouse & PLP peptide & Prophylactic & $=$ & 38 \\
\hline & Systemic (im) & DNA-liposomes & $\mathrm{ABH}$ mouse & $\mathrm{SCH}$ & Prophylactic & $=$ & 34 \\
\hline & Central & DNA-liposomes & $\mathrm{ABH}$ mouse & $\mathrm{SCH}$ & Therapeutic & + & 34 \\
\hline & Systemic (iv) & Vaccinia virus & SJL mouse & $\mathrm{SCH}$ & Prophylactic & $=/-$ & 42 \\
\hline & Central & HSV-1 & BALB/c mouse & $\mathrm{SCH}$ & Prophylactic & + & 50 \\
\hline & Central & HSV-1 & $\mathrm{ABH}$ mouse & MOG peptide & Prophylactic & + & 48 \\
\hline & Central & HSV-1 & $\mathrm{ABH}$ mouse & $\mathrm{SCH}$ & Therapeutic R & + & 51 \\
\hline & Central & HSV-1 & Macaca mulatta & Myelin & Therapeutic & + & 53 \\
\hline & Systemic & RCV-(MBP-T cell) & $(\mathrm{PL} / \mathrm{J} \times \mathrm{SJL})$ mouse & MBP & Therapeutic & + & 56,5 \\
\hline IL-6 & Systemic (iv) & Vaccinia virus & SJL mouse & $\mathrm{SCH}$ & Prophylactic & + & 42 \\
\hline \multirow[t]{12}{*}{ IL-10 } & Systemic (im) & Naked DNA & Lewis rat & MBP peptide & Prophylactic & $=$ & 36 \\
\hline & Systemic (im) & DNA-liposomes & $\mathrm{ABH}$ mouse & $\mathrm{SCH}$ & Prophylactic & $=$ & 34 \\
\hline & Central & DNA-liposomes & $\mathrm{ABH}$ mouse & $\mathrm{SCH}$ & Therapeutic & $=$ & 34,47 \\
\hline & Systemic (v) & Vaccinia virus & SJL mouse & $\mathrm{SCH}$ & Prophylactic & + & 42 \\
\hline & Systemic (iv) & Adenovirus & SJL mouse & $\mathrm{SCH}$ & Prophylactic & $=$ & 46 \\
\hline & Systemic (in) & Adenovirus & SJL mouse & $\mathrm{SCH}$ & Therapeutic & $=$ & 46 \\
\hline & Central & Adenovirus & SJL \& CSJLF1 mouse & $\mathrm{SCH}$ & Therapeutic R & + & 46 \\
\hline & Central & Adenovirus & $\mathrm{ABH}$ mouse & $\mathrm{SCH}$ & Therapeutic & $=$ & 43,47 \\
\hline & Central & HSV-1 & BALB/c mouse & $\mathrm{SCH}$ & Prophylactic & $=$ & 50 \\
\hline & Systemic (iv) & RCV-(PLP-T cell) & (SWR $\times$ SJL) mouse & PLP peptide & Therapeutic & + & 55 \\
\hline & Central & RCV (fibroblast) & $\mathrm{ABH}$ mouse & $\mathrm{SCH}^{1}$ & Therapeutic & + & 47 \\
\hline & Systemic (im) & RCV-(MBP-T cell) & $(\mathrm{PL} / \mathrm{J} \times \mathrm{SJL})$ mouse & $\mathrm{MBP}$ & Therapeutic & $=$ & 56 \\
\hline \multirow[t]{2}{*}{ IFN- $\beta$} & Systemic (im) & DNA-liposomes & $\mathrm{ABH}$ mouse & $\mathrm{SCH}$ & Prophylactic & + & 34 \\
\hline & Central & DNA-liposomes & $\mathrm{ABH}$ mouse & $\mathrm{SCH}$ & Therapeutic & + & 34 \\
\hline \multirow[t]{3}{*}{ IFN- $\gamma$} & Systemic (iv) & Vaccinia virus & SJL mouse & $\mathrm{SCH}$ & Prophylactic & $=$ & 42 \\
\hline & Central & HSV-1 & C57BL/6 mouse & MOG peptide & Prophylactic & + & 52 \\
\hline & Central & HSV-1 & C57BL/6 mouse & MOG peptide & Therapeutic & + & 52 \\
\hline \multirow[t]{4}{*}{ TNF- $\alpha$} & Systemic (im) & Naked DNA & Lewis rat & MBP peptide & Prophylactic & $=$ & 36 \\
\hline & Systemic (im) & DNA-liposomes & $\mathrm{ABH}$ mouse & $\mathrm{SCH}$ & Preventive & $=$ & 34 \\
\hline & Systemic (iv) & Vaccinia virus & SJL mouse & $\mathrm{SCH}$ & Prophylactic & + & 42 \\
\hline & Systemic (iv) & RCV-(MBP-T cell) & (PL/J x SJL) mouse & $\mathrm{MBP}$ & Therapeutic & - & 59,6 \\
\hline \multirow[t]{4}{*}{ p55TNFR-Ig } & Systemic (im) & Naked DNA & $\mathrm{ABH}$ mouse & $\mathrm{SCH}$ & Prophylactic & $=$ & 34 \\
\hline & Systemic (im) & DNA-liposomes & $\mathrm{ABH}$ mouse & $\mathrm{SCH}$ & Prophylactic & + & 34 \\
\hline & Central & DNA-liposomes & $\mathrm{ABH}$ mouse & $\mathrm{SCH}$ & Therapeutic & + & 34 \\
\hline & Central & Adenovirus & $\mathrm{ABH}$ mouse & $\mathrm{SCH}$ & Therapeutic & + & 43 \\
\hline \multirow[t]{4}{*}{ p75TNFR } & Systemic (im) & DNA-liposomes & $\mathrm{ABH}$ mouse & $\mathrm{SCH}$ & Prophylactic & $=$ & 34 \\
\hline & Central & DNA-liposomes & $\mathrm{ABH}$ mouse & $\mathrm{SCH}$ & Therapeutic & + & 34 \\
\hline & Systemic (ip) & RCV (fibroblast) & $\mathrm{ABH}$ mouse & $\mathrm{SCH}$ & Therapeutic & + & 54 \\
\hline & Central & RCV (fibroblast) & $\mathrm{ABH}$ mouse & $\mathrm{SCH}$ & Therapeutic & + & 54 \\
\hline \multirow[t]{4}{*}{ TGF- $\beta$} & Systemic (im) & NAKED DNA & SJL mouse & $\mathrm{MBP}$ & Prophylactic & + & 35 \\
\hline & Systemic (im) & DNA-liposomes & $\mathrm{ABH}$ mouse & $\mathrm{SCH}$ & Prophylactic & $=$ & 34 \\
\hline & Central & DNA-liposomes & $\mathrm{ABH}$ mouse & $\mathrm{SCH}$ & Therapeutic & + & 34 \\
\hline & Systemic (iv) & RCV (PLP-T cell) & $(\mathrm{SJL} \times \mathrm{BALB} / \mathrm{c})$ mouse & PLP peptide & Therapeutic & + & 58 \\
\hline GM-CSF & Systemic (im) & Naked DNA & Lewis rat & MBP peptide & Prophylactic & $=$ & 36 \\
\hline PDGF- $\alpha$ & Systemic (iv) & RCV (PLP-T cell) & (SWR $\times$ SJL) mouse & PLP peptide & Therapeutic & + & 47 \\
\hline FGF & Central & HSV-1 & C57BL/ 6 mouse & MOG peptide & Therapeutic & + & 49 \\
\hline NGF & Systemic (ip) & RCV (MBP-T cell) & Lewis rat & MBP T cell & Prophylactic & + & 61 \\
\hline IP-10 & Systemic (im) & Naked DNA & Lewis rat & MBP peptide & Prophylactic & + & 39 \\
\hline IP-10 & Systemic (im) & Naked DNA & C57BL/6 mouse & MOG peptide & Therapeutic & + & 39 \\
\hline MCP-1 & Systemic (im) & Naked DNA & Lewis rat & MBP T cells & Prophylactic & + & 33 \\
\hline MIP-1 $\alpha$ & Systemic (im) & Naked DNA & Lewis rat & MBP T cells & Prophylactic & + & 33 \\
\hline MIP-1 $\beta$ & Systemic (im) & Naked DNA & Lewis rat & MBP T cells & Prophylactic & - & 33 \\
\hline CAT & Central & Adenoviral & SJL mouse & PLP peptide & Prophylactic & + & 44 \\
\hline NTR p75 & Systemic (ip) & Antisense & SJL/J mouse & PLP peptide & Prophylactic & + & 40 \\
\hline CTLA4Ig & Central & Adenovirus & $\mathrm{ABH}$ mouse & $\mathrm{SCH}$ & Therapeutic & + & 43 \\
\hline $\mathrm{TcRVb}$ & Systemic (im) & Naked DNA & PL mouse & $\mathrm{MBP}$ & Prophylactic & + & 32 \\
\hline PLP epitope & Systemic (im) & Naked DNA & SJL mouse & PLP peptide & Prophylactic & $=$ & 38 \\
\hline PLP \& IL-4 & Systemic (im) & Naked DNA & SJL mouse & PLP peptide & Prophylactic & + & 38 \\
\hline MBP epitope & Systemic (im) & Naked DNA & Lewis rat & MBP peptide & Prophylactic & + & 36,3 \\
\hline MBP \& IL-4 & Systemic (im) & Naked DNA & Lewis rat & MBP peptide & Prophylactic & $=$ & 36 \\
\hline MBP \& IL-10 & Systemic (im) & Naked DNA & Lewis rat & MBP peptide & Prophylactic & $=$ & 36 \\
\hline MOG & Systemic (im) & Naked DNA & C57BL/6 mouse & MOG peptide & Therapeutic R & $=$ & 38 \\
\hline MOG \& IL-4 & Systemic (im) & Naked DNA & C57BL/6 mouse & MOG peptide & Therapeutic R & + & 38 \\
\hline MOG epitope & Systemic (im) & Naked DNA & DA rat & MOG peptide & Prophylactic & + & 41 \\
\hline
\end{tabular}


Table 1 (continued)

\begin{tabular}{|c|c|c|c|c|c|c|c|}
\hline Transgene & Delivery route & Gene vector & Animal strain tested & Inducing antigen & Therapeutic effect & Clinical efficacy & Refs \\
\hline MOG epitope & Systemic (im) & Naked DNA & LEW.1N rat & MOG peptide & Prophylactic & + & 41 \\
\hline PLP epitope & Systemic (iv) & RCV (B cell) & (BALB/c x SJL) mouse & PLP peptide & Prophylactic & + & 62 \\
\hline MBP-Ig & Systemic (iv) & RCV (B cell) & PL $x$ SJL mouse & PLP peptide & Prophylactic & $=$ & 63 \\
\hline MBP-Ig & Systemic (iv) & RCV (B cell) & PL $x$ SJL mouse & MBP peptide & Prophylactic & + & 63 \\
\hline
\end{tabular}

Vectors used in EAE studies contained cytokine transgenes: interleukin (IL), interferon (IFN), tumour necrosis factor (TNF), tumour necrosis factor receptor (TNFR), transforming growth factor (TGF), granulocyte macrophage colony stimulating factor (GM-CSF), platelet-derived growth factor (PDGF), fibroblast growth factor (FGF), nerve growth factor (NGF) and neurotrophin receptor (NTR); Chemokine transgenes: macrophage chemotactic protein (MCP), macrophage inhibitory protein (MIP) and other molecules coding the reactive oxygen species scavenger catalase (CAT), the immunosuppressive CTLA4 immunoglobulin (Ig) fusion protein and myelin antigens. These were administered systemically via the intraperitoneal (ip), intramuscular (im), intranasal (in) or intravenous (iv) routes and were compared with agents injected centrally directly into the CNS, either as naked plasmid DNA, with or without cationic lipids (liposomes) or in adenoviral or herpes simplex viral (HSV) vectors of the transgenes, or were delivered using retrovirally transduced cell vectors (RCV) of fibroblast, B cell, of myelin-specific T-cell origin. These were administered to EAE-susceptible animal strains in either a prophylactic (agent administered before, during or shortly after induction) or therapeutic (shortly before or during development of clinical disease) fashion. In some instances, the gene vectors were delivered in a therapeutic context during relapsing disease (Therapeutic R) and either ameliorated (+), worsened $(-)$ or had no effect of the clinical disease (=).

cells eventually killed the recipient because of unrestricted hybridoma (tumour) growth. ${ }^{56,59,60}$ However, this has been reproduced with ex vivo cloned T cells. ${ }^{55,58}$ In all cases, the T-cell donor cell lines were derived from syngeneic and potentially encephalitogenic T cells (Table 1 ). All healthy individuals appear to harbour peripheral T cells specific for myelin antigens that can be expanded in vitro. ${ }^{1,83,84}$ Therefore, it should be feasible to generate terminally differentiated, nonpathogenic lines that can enter the CNS to release their products. This can be achieved through antigenic selection such as expansion of the $\mathrm{T}$ cells using altered peptide ligands (APL). APL are amino acid-substituted mimics of myelin proteins that drive protective Th2 responses. ${ }^{85-87}$ In EAE, it is clear that autoimmunity to myelin antigens plays an important role in the immune response. ${ }^{1,18,19}$ In humans, there is as yet no definitive proof of obligate autoimmunity to myelin, ${ }^{1,84}$ but using a bystander approach to create an immunosuppressive microenvironment ${ }^{25}$ does not require knowledge of the nature of the pathogenic antigen. However, in vitro preactivation that is a prerequisite for entry of $\mathrm{T}$ cells into the $\mathrm{CNS}^{8,18}$ may induce cytokine release in the blood stream or lymphoid tissues, which may cause side effects. For instance, systemic administration of active recombinant TGF $\beta$ in humans causes reversible nephrotoxicity. ${ }^{27}$ This may have been avoided if the native TGF $\beta$ had been used, as this is typically latent until cleaved at sites of inflammation. It is possible to engineer such cells with suicide genes, so that they can be eliminated should diseaseworsening occur. ${ }^{88}$

\section{Systemic vaccination gene therapy in CNS autoimmunity}

Recent advances in genomics and proteomics have tremendously increased the list of potential targets in $\mathrm{MS}^{16,17,89}$ and may even be used to define autoantigenic targets in individual humans possibly for DNA vaccination reverse genomics. ${ }^{89} \mathrm{~A}$ number of approaches aimed at preventing the generation of encephalitogenic $\mathrm{T}$ cells have been assessed (Table 1)..$^{32,36-38,40,62,63}$ In some EAE models such as the PL/J mouse and Lewis rat, the disease is caused by activity of cells with very limited
T-cell receptor (Tcr) subtype heterogeneity. Here, the majority of encephalitogenic cells express $\mathrm{TcrVb} 8^{90}$ and prophylactic, systemic DNA vaccination against this Tcr subtype has induced EAE amelioration. ${ }^{32}$ While restricted $\mathrm{TcrVb}$ heterogeneity may not always occur, even in inbred animals, ${ }^{91}$ clinical studies in selected patients with repeated $\mathrm{TcrVb}$ peptide injections are already underway and appear safe. ${ }^{92}$ Several studies have targeted the vaccinations to myelin antigens to tolerize the immune response. ${ }^{36-38,62,63}$ This approach should offer the most specific form of therapy and thus limit potential side effects. This has been shown to be active in disease, either as a secreted fusion protein ${ }^{62,63}$ or DNA vaccination, ${ }^{36-38,40}$ induced with all of the three major encephalitogenic myelin proteins (Table 1). In EAE it is clear that different strains respond to different peptide epitopes because of the restrictions imposed by the MHC haplotype(s) expressed, and the pathogenic T-cell repertoire expands with disease progression. ${ }^{1,4}$ The emergence of new T-cell populations generated as a result of previous neurological insult ('determinant spread') is thought, by many, to drive the development of subsequent relapses. ${ }^{4}$ This would limit a simple peptide approach in outbred humans, unless the mechanism of action was bystander suppression, which is not the case in many of the myelin antigen gene therapy studies. ${ }^{37,40,63}$ However, recently it has been shown that determinant spread is not necessary for relapse progression. ${ }^{93}$ This implies that it may be possible to have impact on disease by tolerizing the dominant response that drives relapses, even if minor potentially encephalitogenic responses are present. Gene vaccination studies demonstrate that delivery of whole myelin protein is active at inhibiting EAE. ${ }^{38}$ This suggests that it may be possible to use a cocktail of proteins that could accommodate the majority of epitopes to which a human may respond, especially as the autoantigens in MS are unknown. Furthermore, if bystander suppression can be shown to be the operative mechanism, this may not be necessary. The majority of vaccination studies have only been examined via prophylactic treatment (Table 1). Inhibition of sensitization within lymph nodes can completely prevent the development of disease, but this may not offer any major insight on potential effects, 
possibly worsening the effects of established CNS disease. $^{94,95}$ Further work is required to demonstrate that they are effective in a truly therapeutic context in long-term established disease. However, one study has shown some success in this respect and utilized coadministration of myelin DNA and IL-4 to drive the induction of a Th2 response. ${ }^{38}$ In contrast, a previous study reported that coadministration of IL-4 DNA inhibited the efficacy of myelin DNA vaccination. ${ }^{36} \mathrm{At}$ present DNA vaccination is no more efficient than tolerance induction using a single or short course of native myelin protein. ${ }^{4,95}$ Gene delivery is simpler than the production and application of recombinant proteins and is, therefore, an attractive approach. Although acute EAE is T-cell mediated, ${ }^{1,18}$ myelin-specific B-cell responses contribute to demyelination in relapsing disease $^{96}$ and evidence from rodent and primate studies suggest that a Th2-deviated B-cell response may even exacerbate chronic disease. ${ }^{97,98}$ Repeated subcutaneous injections of myelin-altered peptide ligands in MS were halted because of the occurrence of adverse effects. ${ }^{99,100}$ These effects were not evident in acute EAE, where essentially all of the gene therapy studies have been performed, but were subsequently shown to occur in rodents following repeated administration of peptide in established relapsing EAE. ${ }^{97}$ Therefore, it will be important to demonstrate the efficacy of DNA vaccination in long-established EAE and suggests also that gene expression needs to be controlled in the clinic.

\section{Future prospects for gene therapy in CNS autoimmune demyelination}

Although MS is considered to be an immune-mediated disease, immunosuppressive therapies currently fail to inhibit disease progression. ${ }^{2,26,101}$ However, these therapies have had positive outcomes in reducing lesion formation and the relapse rate. ${ }^{2,26,101}$ This is beginning to underscore the new belief that the major cause of permanent progressive disability is because of neurodegeneration. ${ }^{102,103}$ While this occurs early in MS and EAE, ${ }^{102,103}$ it is likely that because of inflammationinduced demyelination, a CNS microenvironment is created where the nerves are particularly sensitive to neurotoxic insults, such as glutamate excitotoxcity, oxidative free-radical damage and toxic ion fluxes, and a slow degenerative process that may continue independent of the inflammatory response is triggered. ${ }^{102,104-107}$ Although some success has been shown by genedelivered growth factors that provide trophic support (eg NGF and PDGF. Table 1), 49,57 neuroprotection by any route in CNS autoimmunity, particularly in long-established disease, is essentially unexplored. However, there is evidence from other neurodegenerative systems that gene delivery of growth factors can promote remyelination. ${ }^{108-110}$ It is likely that effective therapy will require a combination of agents that target different elements of the disease process (Figure 1). At the moment, monotherapies are largely being investigated and these have concentrated on targeting the immune response. ${ }^{26}$ Therefore, perhaps our expectations of a treatment are too high and success will probably only be realized once a combination of therapies are applied. Gene therapy may easily allow multiple products to be delivered that can target different pathways; however, much more work is required to show that these can work and importantly that they are safe. MS is a chronic disease and although the quality of life is reduced, it is not rapidly fatal. ${ }^{1,2}$ Therefore, any agents should be used cautiously and safely. It is likely that CNS-directed gene therapy would be clinically developed in other more fatal diseases such as brain tumours where some clinical studies have been undertaken. ${ }^{111}$ Gene vectors will need to be inducible and ideally removable, as clinical experience has been found that some animal studies do not translate and treatments may make disease worse. ${ }^{112,113}$ Although there is supportive evidence that MS is a Th1-mediated disease, ${ }^{1,2,114}$ the Th1/Th2 paradigm is less clear-cut in humans. While Th2 deviation may be part of the action of IFN $\beta,{ }^{115}$ further studies are warranted, as it is important to determine whether a Th2-deviated response is safe in long-established disease. In 'classical' EAE, while CD4 encephalitogenic cells are Th1, IFN $\gamma$ has consistently been shown to be protective, ${ }^{113,116}$ and thus contrasts with observations in humans. ${ }^{117,118}$ However, recently a CD8-mediated disease has been induced in which IFN $\gamma$ is proinflammatory. ${ }^{119,120}$ In human MS, there is evidence for CD8 T-cell expansions within the CNS, ${ }^{121,122}$ which is indicative of local immune activity and further studies are warranted in this area as it is important that the models reflect events occurring in human disease. Furthermore, inhibition of TNF activity has consistently demonstrated disease-ameliorating effects in EAE although disease worsening occurred following systemic TNF neutralization in MS. ${ }^{29}$ This has also been observed in some EAE systems. ${ }^{112}$ Cytokines regulate the immune response and systemic perturbation of the homeostatic balance can induce pro or anti-inflammatory effects. Animal studies suggest that local targeting of proinflammatory cytokines within the target tissue is important, yet this has seldom been attempted in humans and is a challenge for the future. At the moment, gene therapy is a useful experimental tool, which can help dissect mechanisms in experimental disease but it is still in its infancy. While it offers future promise, much has to be done before this can be considered as a therapy in human CNS autoimmunity.

\section{Acknowledgements}

The Multiple Sclerosis Society of Great Britain and Northern Ireland supported our work.

\section{References}

1 Raine CS, McFarland HF, Tourtellotte WW (eds). Multiple Sclerosis. Clinical and Pathogenetic Basis. Chapman \& Hall: London, 1997.

2 Compston A, Coles A. Multiple sclerosis. Lancet. 2002; 359: 12211231. MEDLINE

3 Compston A, Sawcer S. Genetic analysis of multiple sclerosis. Curr Neurol Neurosci Rep 2002; 2: 259-266. MEDLINE

4 Vanderlugt CL, Miller SD. Epitope spreading in immunemediated diseases: implications for immunotherapy. Nat Rev Immunol 2002; 2: 85-95. MEDLINE. 
5 Olson JK, Eagar TN, Miller SD. Functional activation of myelinspecific $\mathrm{T}$ cells by virus-induced molecular mimicry. J Immunol 2002; 169: 2719-2726. MEDLINE.

6 van Noort JM, Bajramovic JJ, Plomp AC, van Stipdonk MJ. Mistaken self, a novel model that links microbial infections with myelin-directed autoimmunity in multiple sclerosis. J Neuroimmunol. 2000; 105: 46-57. MEDLINE

7 Alvord ECJ, Kies MW, Suckling AJ (eds). Experimental Allergic Encephalomylitis: A Useful Model For Multiple Sclerosis. Allen R Liss: New York, 1984.

8 Flugel A et al. Migratory activity and functional changes of green fluorescent effector cells before and during experimental autoimmune encephalomyelitis. Immunity. 2001; 14: 547-560. MEDLINE

9 Butter C, Baker D, O'Neill JK, Turk JL. Mononuclear cell trafficking and plasma protein extravasation into the CNS during chronic relapsing experimental allergic encephalomyelitis in Biozzi AB/H mice. J Neurol Sci. 1991; 104: 9-12. MEDLINE

10 Karin $\mathrm{N}$ et al. Selective and nonselective stages in homing of $\mathrm{T}$ lymphocytes to the central nervous system during experimental allergic encephalomyelitis. J Immunol 1993; 150: 4116-4124. MEDLINE

11 Steinman L et al. Multiple sclerosis: deeper understanding of its pathogenesis reveals new targets for therapy. Annu Rev Neurosci. 2002; 25: 491-505. MEDLINE.

12 Baker D, Gibjels K, Steinman LS. Cytokines in multiple sclerosis. In: The Role of Cytokines in Autoimmunity. Brennan FO, Feldmann M (eds). RG Landes Company: Austin, 1996. pp 77-99.

13 Issazadeh $S$ et al. Cytokine production in the central nervous system of Lewis rats with experimental autoimmune encephalomyelitis: dynamics of mRNA expression for interleukin-10, interleukin-12, cytolysin, tumor necrosis factor alpha and tumor necrosis factor beta. J Neuroimmunol 1995; 61: 205-212. Article MEDLINE

14 Kennedy MK, Torrance DS, Picha KS, Mohler KM. Analysis of cytokine mRNA expression in the central nervous system of mice with experimental autoimmune encephalomyelitis reveals that IL-10 mRNA expression correlates with recovery. J Immunol 1992; 149: 2496-2505. MEDLINE

15 Baranzini SE et al. Transcriptional analysis of multiple sclerosis brain lesions reveals a complex pattern of cytokine expression. $J$ Immunol. 2000; 165: 6576-6582. MEDLINE

16 Lock $\mathrm{C}$ et al. Gene-microarray analysis of multiple sclerosis lesions yields new targets validated in autoimmune encephalomyelitis. Nat Med 2002; 8: 500-508. MEDLINE

17 Romagnani S. T-cell subsets (Th1 versus Th2). Ann Allergy Asthma Immunol. 2000; 85: 9-18. MEDLINE

18 Ando DG et al. Encephalitogenic T cells in the B10.PL model of experimental allergic encephalomyelitis (EAE) are of the Th-1 lymphokine subtype. Cell Immunol 1989; 124: 132-143. MEDLINE

19 Youssef $\mathrm{S}$ et al. The HMG-CoA reductase inhibitor, atorvastatin, promotes a Th2 bias and reverses paralysis in central nervous system autoimmune disease. Nature 2002; 420: 78-84. MEDLINE

20 Brok HP et al. Non-human primate models of multiple sclerosis. Immunol Rev. 2001; 183: 173-185. MEDLINE

21 Leonard JP, Waldburger KE, Goldman SJ. Prevention of experimental autoimmune encephalomyelitis by antibodies against interleukin 12. J Exp Med 1995; 181: 381-386. MEDLINE

22 Falcone M, Rajan A, Bloom B, Brosnan C. A critical role for IL-4 in regulating disease severity in experimental allergic encephalomyelitis as demonstrated in IL-4 deficient C57BL/6 and BALB/c mice. J Immunol 1998; 160: 4822-4830. MEDLINE

23 Rott O, Fleischer B, Cash E. Interleukin-10 prevents experimental allergic encephalomyelitis in rats. Eur J Immunol 1994; 24: 14341440. MEDLINE

24 Samoilova E, Horton J, Chen Y. Acceleration of experimental autoimmune encephalomyelitis in interleukin-10-deficient mice: roles of interleukin-10 in disease progression and recovery. Cell Immunol 1998; 188: 118-124. MEDLINE

25 Chen $\mathrm{Y}$ et al. Regulatory T-cell clones induced by oral tolerance: suppression of autoimmune encephalomyelitis. Science 1994; 265: 1237-1240. MEDLINE

26 Wiendl H, Hohlfeld R. Therapeutic approaches in multiple sclerosis: lessons from failed and interrupted treatment trials. BioDrugs 2002; 16: 183-200. MEDLINE

27 Calabresi PA et al. Phase 1 trial of transforming growth factor beta 2 in chronic progressive MS. Neurology 1998; 51: 289-292. MEDLINE

28 The IFNB Multiple Sclerosis Study Group and the University of British Columbia MS/MRI Analysis Group. Neutralizing antibodies during treatment of multiple sclerosis with interferon beta-1b: experience during the first three years. Neurology 1996; 47: 889-894. MEDLINE

29 The Lenercept Multiple Sclerosis Study Group and University of British Columbia MS/MRI Analysis Group. TNF neutralization in MS: results of a randomized, placebo-controlled multicenter study. Neurology 1999; 53: 457-465.

30 van Oosten BW et al. Increased MRI activity and immune activation in two multiple sclerosis patients treated with the monoclonal anti-tumor necrosis factor antibody cA2. Neurology 1996; 7: 1531-1534.

31 Saunders NR, Habgood MD, Dziegielewska KM. Barrier mechanisms in the brain, I. Adult brain. Clin Exp Pharmacol Physiol 1999; 26: 11-19. MEDLINE

32 Waisman A et al. Suppressive vaccination with DNA encoding a variable region gene of the T-cell receptor prevents autoimmune encephalomyelitis and activates Th2 immunity. Nature Med 1996; 2: 899-905. MEDLINE

33 Youssef $\mathrm{S}$ et al. Long lasting protective immunity to experimental autoimmune encephalomyelitis following vaccination with naked DNA encoding C-C chemokines. J Immunol 1998; 161: 3870-3879. MEDLINE

34 Croxford JL et al. Cytokine gene therapy in experimental allergic encephalomyelitis by injection of plasmid DNA-cationic liposome complex into the central nervous system. J Immunol 1998; 160: 5181-5187. MEDLINE

35 Piccirillo CA, Prud'homme GJ. Prevention of experimental allergic encephalomyelitis by intramuscular gene transfer with cytokine-encoding plasmid vectors. Hum Gene Ther 1999; 10: 1915-1922. MEDLINE

36 Lobell A et al. Presence of CpG DNA and the local cytokine milieu determine the efficacy of suppressive DNA vaccination in experimental autoimmune encephalomyelitis. J Immunol 1999; 163: 4754-4762. MEDLINE

37 Weissert $\mathrm{R}$ et al. Protective DNA vaccination against organspecific autoimmunity is highly specific and discriminates between single amino acid substitutions in the peptide autoantigen. Proc Natl Acad Sci USA 2000; 97: 1689-1694. MEDLINE

38 Garren $\mathrm{H}$ et al. Combination of gene delivery and DNA vaccination to protect from and reverse Th1 autoimmune disease via deviation to the Th2 pathway. Immunity 2001; 15: 15-22. MEDLINE

39 Wildbaum G, Netzer N, Karin N. Plasmid DNA encoding IFN-gamma-inducible protein 10 redirects antigen-specific T-cell polarization and suppresses experimental autoimmune encephalomyelitis. J Immunol 2002; 168: 5885-5892. MEDLINE

40 Lobell A et al. Suppressive DNA Vaccination in myelin oligodendrocyte glycoprotein peptide-induced experimental autoimmune encephalomyelitis involves a T1-biased immune response. J Immunol 2003; 170: 1806-1813. MEDLINE

41 Soilu-Hanninen $\mathrm{M}$ et al. Treatment of experimental autoimmune encephalomyelitis with antisense oligonucleotides against the low affinity neurotrophin receptor. J Neurosci Res 2000; 59: 712721. MEDLINE 
42 Willenborg DO, Fordham SA, Cowden WB, Ramshaw IA. Cytokines and murine autoimmune encephalomyelitis: inhibition or enhancement of disease with antibodies to select cytokines, or by delivery of exogenous cytokines using a recombinant vaccinia virus system. Scand J Immunol 1995; 41: 31-41. MEDLINE

43 Croxford JL et al. Local gene therapy with CTLA4-immunoglobulin fusion protein in experimental allergic encephalomyelitis. Eur I Immunol 1998; 28: 3904-3916. MEDLINE

44 Guy J, Qi X, Wang H, Hauswirth WW. Adenoviral gene therapy with catalase suppresses experimental optic neuritis. Arch Ophthalmol 1999; 117: 1533-1539. MEDLINE

45 Kawaguchi Y. A gene therapy or purified CTLA4IgG treatment of experimental allergic encephalomyelitis. Hokkaido Igaku Zasshi 1999; 74: 467-475. MEDLINE

46 Cua DJ et al. Central nervous system expression of IL-10 inhibits autoimmune encephalomyelitis. J Immunol 2001; 166: 602-608. MEDLINE

47 Croxford JL, Feldmann M, Chernajovsky Y, Baker D. Different therapeutic outcomes in experimental allergic encephalomyelitis dependent upon the mode of delivery of IL-10: a comparison of the effects of protein, adenoviral or retroviral IL-10 delivery into the central nervous system. J Immuno 2001; 166: 4124-4130. MEDLINE

48 Furlan $\mathrm{R}$ et al. Central nervous system delivery of interleukin- 4 by a non-replicative herpes simplex type 1 viral vector ameliorates autoimmune demyelination. Hum Gene Ther 1998; 9: 2605-2617. MEDLINE

49 Ruffini $\mathrm{F}$ et al. Fibroblast growth factor-II gene therapy reverts the clinical course and the pathological signs of chronic experimental autoimmune encephalomyelitis in C57BL/6 mice. Gene Ther. 2000; 8: 1207-1213. MEDLINE

50 Broberg E et al. Expression of interleukin-4 but not of interleukin-10 from a replicative herpes simplex virus type 1 viral vector precludes experimental allergic encephalomyelitis. Gene Ther. 2001; 8: 769-777. MEDLINE

51 Furlan $\mathrm{R}$ et al. Central nervous system gene therapy with interleukin- 4 inhibits progression of ongoing relapsing-remitting autoimmune encephalomyelitis in Biozzi $\mathrm{AB} / \mathrm{H}$ mice. Gene Ther 2001; 8: 13-19. MEDLINE

52 Furlan $\mathrm{R}$ et al. Intrathecal delivery of IFN-gamma protects C57BL/6 mice from chronic-progressive experimental autoimmune encephalomyelitis by increasing apoptosis of central nervous system-infiltrating lymphocytes. J Immunol 2001; 167: 1821-1829. MEDLINE

53 Poliani PL et al. Delivery to the central nervous system of a nonreplicative herpes simplex type 1 vector engineered with the interleukin 4 gene protects rhesus monkeys from hyperacute autoimmune encephalomyelitis. Hum Gene Ther 2001; 12: 905920. MEDLINE

54 Croxford JL et al. Gene therapy for chronic relapsing experimental allergic encephalomyelitis using cells expressing a novel soluble p75 dimeric TNF receptor. I Immunol 2001; 164: 27762781. MEDLINE

55 Mathisen PM et al. Treatment of experimental autoimmune encephalomyelitis with genetically modified memory T cells. $J$ Exp Med 1997; 186: 159-164. MEDLINE

56 Shaw MK et al. Local delivery of interleukin 4 by retrovirustransduced $\mathrm{T}$ lymphocytes ameliorates experimental autoimmune encephalomyelitis. J Exp Med 1997; 185: 1711-1714. MEDLINE

57 Mathisen PM et al. Th2 T cells expressing transgene PDGF-A serve as vectors for gene therapy in autoimmune demyelinating disease. J Autoimmun 1999; 13: 31-38. MEDLINE

58 Chen LZ et al. Gene therapy in allergic encephalomyelitis using myelin basic protein-specific T cells engineered to express latent transforming growth factor-ß1. Proc Natl Acad Sci USA 1998; 95: 12516-12521. MEDLINE
59 Dal Canto RA et al. Local delivery of cytokines by retrovirally transduced antigen-specific TCR hybridoma cells in experimental autoimmune encephalomyelitis. Eur Cytokine Netw 1998; 9: 83-91. MEDLINE

60 Dal Canto RA et al. Local delivery of TNF by retrovirustransduced $\mathrm{T}$ lymphocytes exacerbates experimental autoimmune encephalomyelitis. Clin Immunol 1999; 90: 10-14. MEDLINE

61 Flugel A et al. Anti-inflammatory activity of nerve growth factor in experimental autoimmune encephalomyelitis: inhibition of monocyte transendothelial migration. Eur J Immunol 2001; 31: 11-22. MEDLINE

62 Chen $\mathrm{C}$ et al. A gene therapy approach for treating T-cellmediated autoimmune diseases. Blood 2001; 97: 886-894. MEDLINE

63 Melo ME et al. Gene transfer of Ig-fusion proteins into B cells prevents and treats autoimmune diseases. J Immunol 2002; 168: 4788-4795. MEDLINE

64 Martino G, Furlan R, Comi G, Adorini L.The ependymal route to the CNS: an emerging gene-therapy approach for MS. Trends Immunol 2001; 22: 483-490. MEDLINE

65 Becher B, Durell BG, Noelle RJ. Experimental autoimmune encephalitis and inflammation in the absence of interleukin-12. J Clin Invest 2002; 110: 493-497. MEDLINE

66 Schapiro RT. M anagement of spasticity, pain, and paroxysmal phenomena in multiple sclerosis. Curr Neurol Neurosci Rep 2001; 1: 299-302. MEDLINE

67 Martino G et al. Cytokine therapy in immune-mediated demyelinating diseases of the central nervous system: a novel gene therapy approach. J Neuroimmunol 2000; 107: 184-190. MEDLINE

68 Allen SJ et al. Isolation and characterization of cells infiltrating the spinal cord during the course of chronic relapsing experimental allergic encephalomyelitis in the Biozzi $\mathrm{AB} / \mathrm{H}$ mouse. Cell Immunol 1993; 146: 335-350. MEDLINE

69 Boccaccio GL, Mor F, Steinman L. Noncoding plasmid DNA induces IFN- $\gamma$ in vivo and suppresses autoimmune encephalomyelitis. Int Immunol 1999; 11: 289-296. MEDLINE

70 Tsunoda I et al. Exacerbation of viral and autoimmune animal models for multiple sclerosis by bacterial DNA. Brain Pathol 1999; 9: 481-493. MEDLINE

71 Shimeld $C$ et al. Cytokine production in the nervous system of mice during acute and latent infection with herpes simplex virus type 1. J Gen Virol 1997; 78: 3317-3325. MEDLINE

72 Modo M et al. Transplantation of neural stem cells in a rat model of stroke: assessment of short-term graft survival and acute host immunological response. Brain Res 2002; 958: 70-82. MEDLINE

73 Thomas CE et al. Peripheral infection with adenovirus causes unexpected long-term brain inflammation in animals injected intracranially with first-generation, but not with high-capacity, adenovirus vectors: toward realistic long-term neurological gene therapy for chronic diseases. Proc Natl Acad Sci USA 2000; 97: 7482-7487. MEDLINE

74 Ali RR et al. Co-injection of adenovirus expressing CTLA4-Ig prolongs adenovirally mediated lac $Z$ reporter gene expression in the mouse retina. Gene Ther 1998; 5: 1561-1565. MEDLINE

75 Lowenstein PR. Immunology of viral-vector-mediated gene transfer into the brain: an evolutionary and developmental perspective. Trends Immunol 2002; 23: 23-30. M . MEDLINE

76 Cao Q, Benton RL, Whittmore SR. Stem cell repair of central nervous system injury. J Neurosci Res 2002; 68: 501-510. MEDLINE

77 Dezawa M. Central and peripheral nerve regeneration by transplantation of Schwann cells and transdifferentiated bone marrow stromal cells. Anat Sci Int 2002; 77: 12-25. MEDLINE 
78 Galvin KA, Jones DG. Adult human neural stem cells for cellreplacement therapies in the central nervous system. Med J Aust 2002; 177: 316-318. MEDLINE

79 Franklin RJ. Remyelination of the demyelinated CNS: the case for and against transplantation of central, peripheral and olfactory glia. Brain Res Bull 2002; 57: 827-832. MEDLINE

80 Chari DM, Blakemore WF.New insights into remyelination failure in multiple sclerosis: implications for glial cell transplantation. Mult Scler 2002; 8: 271-277. MEDLINE

81 Aebischer $\mathrm{P}$ et al. Intrathecal delivery of CNTF using encapsulated genetically modified xenogeneic cells in amyotrophic lateral sclerosis patients. Nat Med 1996; 2: 696-699. MEDLINE

82 Yura $\mathrm{M}$ et al. Role of MOG-stimulated Th1 type 'light up' (GFP+) CD4+ T cells for the development of experimental autoimmune encephalomyelitis (EAE). J Autoimmun 2001; 17: 17-25. MEDLINE

83 Pette $\mathrm{M}$ et al. $\mathrm{M}$ yelin basic protein-specific $\mathrm{T}$ lymphocyte lines from MS patients and healthy individuals. Neurology 1990; 40: 1770-1776. MEDLINE

84 van Noort JM et al. The small heat-shock protein alpha Bcrystallin as candidate autoantigen in multiple sclerosis. Nature 1995; 375: 798-801. MEDLINE

85 Nicholson LB et al. An altered peptide ligand mediates immune deviation and prevents autoimmune encephalomyelitis. Immunity 1995; 3: 397-405. MEDLINE

86 Young DA et al. IL-4, IL-10, IL-13, and TGF-beta from an altered peptide ligand-specific Th2 cell clone down-regulate adoptive transfer of experimental autoimmune encephalomyelitis. $J$ Immunol 2000; 164: 3563-3572. MEDLINE

$87 \mathrm{Kim} \mathrm{HJ}$ et al. Persistence of immune responses to altered and native myelin antigens in patients with multiple sclerosis treated with altered peptide ligand. Clin Immunol 2002; 104: 105-114. MEDLINE

88 Chernajovsky $\mathrm{Y}$ et al. Inhibition of transfer of collagen-induced arthritis into SCID mice by ex vivo infection of spleen cells with retroviruses expressing soluble tumor necrosis factor receptor. Gene Ther 1995; 2: 731-735.

89 Robinson WH, Garren H, Utz PJ, Steinman L. Millennium Award. Proteomics for the development of DNA tolerizing vaccines to treat autoimmune disease. Clin Immunol 2002; 103: 712. MEDLINE

90 Acha-Orbea et al. Limited heterogeneity of $\mathrm{T}$ cell receptors from lymphocytes mediating autoimmune encephalomyelitis allows specific immune intervention. Cell 1988; 54: 263-273. MEDLINE

91 Kuchroo VK et al. Experimental allergic encephalomyelitis mediated by cloned $\mathrm{T}$ cells specific for a synthetic peptide of myelin proteolipid protein. Fine specificity and $\mathrm{T}$ cell receptor $\mathrm{V}$ beta usage. J Immunol 1992; 148: 3776-3782. MEDLINE

92 Vandenbark AA et al. TCR peptide therapy in human autoimmune diseases. Neurochem Res 2001; 26: 713-730. MEDLINE

93 Jones RE et al. Epitope spreading is not required for relapses in experimental autoimmune encephalomyelitis. J Immunol 2003; 170: 1690-1698. MEDLINE

$94 \mathrm{O}^{\prime}$ Neill JK et al. Therapy of chronic relapsing experimental allergic encephalomyelitis and the role of the blood-brain barrier: elucidation by the action of Brequinar sodium. $J$ Neuroimmunol 1992; 38: 53-62. MEDLINE

95 O'Neill JK, Baker D, Turk JL. Inhibition of chronic relapsing experimental allergic encephalomyelitis in the Biozzi $\mathrm{AB} / \mathrm{H}$ mouse. J Neuroimmunol 1992; 41: 177-187. MEDLINE

96 Morris-Downes MM et al. Pathological and regulatory effects of anti-myelin antibodies in experimental allergic encephalomyelitis in mice. J Neuroimmunol 2002; 125: 114-124. MEDLINE

97 Pedotti $\mathrm{R}$ et al. An unexpected version of horror autotoxicus: anaphylactic shock to a self-peptide. Nat Immunol 2001; 2: 216222. MEDLINE
98 Genain CP et al. Late complications of immune deviation therapy in a nonhuman primate. Science 1996; 274: 2054-2057. MEDLINE

99 Bielekova B et al. Encephalitogenic potential of the myelin basic protein peptide (amino acids 83-99) in multiple sclerosis: results of a phase II clinical trial with an altered peptide ligand. Nat Med 2000; 6: 1167-1175. MEDLINE

100 Kappos L et al. Induction of a non-encephalitogenic type $2 \mathrm{~T}$ helper-cell autoimmune response in multiple sclerosis after administration of an altered peptide ligand in a placebocontrolled, randomized phase II trial. The Altered Peptide Ligand in Relapsing MS Study Group. Nat Med 2000; 6: 11761182. MEDLINE

101 Coles AJ et al. M onoclonal antibody treatment exposes three mechanisms underlying the clinical course of multiple sclerosis. Ann Neurol 1999; 46: 296-304. MEDLINE

102 Bjartmar C, Wujek JR, Trapp BD. Axonal loss in the pathology of MS: consequences for understanding the progressive phase of the disease. J Neurol Sci 2003; 206: 165-171. MEDLINE

103 Wujek JR et al. Axon loss in the spinal cord determines permanent neurological disability in an animal model of multiple sclerosis. J Neuropathol Exp Neurol 2002; 61: 23-32. MEDLINE

104 Werner P, Pitt D, Raine CS. Multiple sclerosis: altered glutamate homeostasis in lesions correlates with oligodendrocyte and axonal damage. Ann Neurol 2001; 50: 169-180. MEDLINE

105 Pitt D, Werner P, Raine CS. Glutamate excitotoxicity in a model of multiple sclerosis. Nat Med 2000; 6: 67-70. MEDLINE

106 Lo AC, Black JA, Waxman SG. Neuroprotection of axons with phenytoin in experimental allergic encephalomyelitis. Neuroreport 2002; 13: 1909-1912. MEDLINE

107 Kapoor $\mathrm{R}$ et al. Blockers of sodium and calcium entry protect axons from nitric oxide-mediated degeneration. Ann Neurol 2003; 53: 174-180. MEDLINE

108 Stankoff B et al. Ciliary neurotrophic factor (CNTF) enhances myelin formation: a novel role for CNTF and CNTF-related molecules. J Neurosci 2002; 22: 9221-9227. MEDLINE

109 Wang LJ et al. Neuroprotective effects of glial cell line-derived neurotrophic factor mediated by an adeno-associated virus vector in a transgenic animal model of amyotrophic lateral sclerosis. J Neurosci 2002; 22: 6920-6928.

110 Alberch J, Perez-Navarro E, Canals JM. Neuroprotection by neurotrophins and GDNF family members in the excitotoxic model of Huntington's disease. Brain Res Bull 2002; 57: 817-822. MEDLINE

111 Markert J et al. Conditionally replicating herpes simplex virus mutant, G207 for the treatment of malignant glioma: results of a phase I trial. Gene Therapy 2000; 7: 867-874. MEDLINE

112 Lock C, Oksenberg J, Steinman L. The role of TNF alpha and lymphotoxin in demyelinating disease. Ann Rheum Dis 58(Suppl 1): I121-I128. MEDLINE

113 Steinman L. M yelin-specific CD8 T cells in the pathogenesis of experimental allergic encephalitis and multiple sclerosis. J Exp Med 2001; 194: F27--F30. MEDLINE

114 Correale $\mathrm{J}$ et al. Patterns of cytokine secretion by autoreactive proteolipid protein-specific $\mathrm{T}$ cell clones during the course of multiple sclerosis. J Immunol 1995; 154: 2959-2968. MEDLINE

115 Neuhaus $\mathrm{O}$ et al. Statins as immunomodulators: comparison with interferon-beta 1b in MS. Neurology 2002; 59: 990-997. MEDLINE

$116 \mathrm{Chu} C Q$, Wittmer S, Dalton DK. Failure to suppress the expansion of the activated CD4 T cell population in interferon gamma-deficient mice leads to exacerbation of experimental autoimmune encephalomyelitis. J Exp Med 2000; 192: 123-128. MEDLINE

117 Panitch NL, Hirsch RL, Haley AS, Johnson KP. Exacerbations of multiple sclerosis in patients treated with gamma interferon. Lancet 1987; 1: 893-895. MEDLINE 
118 Skurkovich S. Randomized study of antibodies to IFN-gamma and TNF-alpha in secondary progressive multiple sclerosis. Mult Scler 2001; 7: 277-2784. MEDLINE

119 Hueseby ES et al. A pathogenic role for myelin-specific CD8(+) $\mathrm{T}$ cells in a model for multiple sclerosis. J Exp Med 2001; 194: 669-676. MEDLINE

120 Sun $\mathrm{D}$ et al. $\mathrm{M}$ yelin antigen-specific CD8+ $\mathrm{T}$ cells are encephalitogenic and produce severe disease in C57BL/6 mice. I Immunol 2001; 166: 7579-7587. MEDLINE
121 Babbe $\mathrm{H}$ et al. clonal expansions of $\mathrm{CD} 8(+) \mathrm{T}$ cells dominate the $\mathrm{T}$ cell infiltrate in active multiple sclerosis lesions as shown by micromanipulation and single cell polymerase chain reaction. $J$ Exp Med 2000; 192: 393-404. MEDLINE

122 Jacobsen $\mathrm{M}$ et al. Oligoclonal expansion of memory CD8+ $\mathrm{T}$ cells in cerebrospinal fluid from multiple sclerosis patients. Brain 2002; 125: 538-550. MEDLINE 